\title{
Correction to: Dynamics for a Three-Species Predator-Prey Model with Density-Dependent Motilities
}

\author{
Shuyan Qiu ${ }^{1} \cdot$ Chunlai $\mathrm{Mu}^{2} \cdot$ Xinyu $\mathrm{Tu}^{3}$ \\ Published online: 17 September 2021 \\ ○ Springer Science+Business Media, LLC, part of Springer Nature 2021
}

\section{Correction to: Journal of Dynamics and Differential Equations https://doi.org/10.1007/s10884-021-10020-6}

The original version of this article unfortunately contained error in Acknowledgement section. Therefore, the correct and complete section is given here:

Acknowledgements The first author is supported by Young scholars development fund of SWPU grant 202199010087. The second author is supported by NSFC under grants 11771062 and 11971082, the Fundamental Research Funds for the Central Universities under grants 2019CDJCYJ001, 2020CDJQY-Z001 and XDJK2020C054, Chongqing Key Laboratory of Analytic Mathematics and Applications. The third author is supported by China Postdoctoral Science Foundation under Grant 2020M673102, the Natural Science Foundation of Chongqing, China under grant cstc2020jcyj-bshX0071, Chongqing Post-doctoral Innovative Talent Support program.

The original article has been corrected.

Publisher's Note Springer Nature remains neutral with regard to jurisdictional claims in published maps and institutional affiliations.

The original article can be found online at https://doi.org/10.1007/s10884-021-10020-6.

Shuyan Qiu

shuyanqiu0701@126.com

Chunlai $\mathrm{Mu}$

clmu2005@163.com

1 School of Sciences, Southwest Petroleum University, Chengdu 610500, People's Republic of China

2 College of Mathematics and Statistics, Chongqing University, Chongqing 401331, People's Republic of China

3 School of Mathematics and Statistics, Southwest University, Chongqing 400715, People's Republic of China 